\title{
Satellite remote sensing in earthquake prediction. A review
}

\begin{abstract}
Sudden, violent movement of the earth's surface resulted of the release of energy into the atmosphere can destroy cities and claim lives. With the recent advances in space-borne data collecting methods which have made it possible monitoring the earth surface with different sensors, scientists are now able to better study the causes and signs of Earthquakes. Current researches are moving in the direction of pre-earthquake deformation detection. In order to make successful prediction all the related data must be collected from different space-borne sensors and ground-based stations. Past earthquakes should also be investigated for any phenomena that can occur before an earthquake. Surface deformation data are provided by GPS and SAR imaging, land surface temperature changes by ASTER, Landsat TM and ETM, different types of cloud studies using MODIS and Seawifs data, electromagnetic and ionosphere anomalies by ground passive stations and radon gas emissions in the faults areas by solid on the ground detectors. In this paper we tried to gather complete and helpful information of earthquake precursors which have been studied until now.
\end{abstract}

Keyword: Natural hazard; Earthquake prediction; Earthquake precursors; Remote sensing; Ground-based monitoring 